\title{
A comparison of trauma scoring systems for trauma-related injuries presenting to a district- level urban public hospital in Western Cape, South Africa
}

\author{
SN Mukonkole, ${ }^{1,2}$ L Hunter, ${ }^{2}$ A Möller, ${ }^{3}$ M Mccaul, ${ }^{4}$ S Lahri, ${ }^{2}$ DJ Van Hoving ${ }^{5}$ \\ ${ }^{I}$ Division of Epidemiology and Biostatistics, Global health, Stellenbosch University, Western Cape, South Africa \\ ${ }^{2}$ Khayelitsha Hospital, Western Cape, South Africa \\ ${ }^{3}$ Karonlinska Institute, Sweden \\ ${ }^{4}$ Division of Epidemiology and Biostatistics, Global Health, Stellenbosch University, Western Cape, South Africa \\ ${ }^{5}$ Division of Emergency Medicine, Stellenbosch University, Western Cape, South Africa
}

Corresponding author, email: smukonkole@gmail.com

\begin{abstract}
Background: Trauma is a major public health issue and has an extensive burden on the health system in South Africa. Many trauma scoring systems have been developed to estimate trauma severity and predict mortality. The prediction of mortality between different trauma scoring systems have not been compared at district-level health facilities in South Africa. The objective was to compare four trauma scoring systems (injury severity score (ISS), revised trauma score (RTS), Kampala trauma score (KTS), trauma and injury severity score (TRISS)) in predicting mortality in trauma-related patients presenting to a district-level hospital in Cape Town.

Methods: A retrospective analysis of all trauma patients managed in the resuscitation unit of Khayelitsha Hospital during a six-month period. Logistic regression was done, and empirical cut of points used to maximise sensitivity and specificity on receiver operating characteristic curves. The outcome was all-cause in-hospital mortality.

Results: In total, 868 participants were analysed after 50 were excluded due to missing data. The mean ( \pm SD) age was $28 \pm 11$ years, $726(83.6 \%)$ were males, and penetrating injuries $(n=492,56.6 \%)$ dominated. The mortality rate was $5.2 \%$ $(\mathrm{n}=45)$. TRISS was the best mortality predictor (c-statistic 0.93 , sensitivity $90 \%$, specificity $87 \%$ ). All scoring systems had overlapping confidence intervals.

Conclusion: TRISS, ISS, RTS and KTS performed equivocally in predicting mortality in trauma-related patients managed at a district-level facility. The appropriate scoring system should be the simplest one which can be practically implemented and will likely differ between facilities.
\end{abstract}

S Afr J Surg 2020;58(1)

http://dx.doi.org/10.17159/2078-5151/2020/v58n1a3116

\section{Introduction}

Trauma is a global health problem and arguably affects lowand middle-income countries disproportionally more. Trauma accounts for $15 \%$ of deaths in Sub-Saharan Africa and for $11 \%$ of deaths in South Africa. ${ }^{1}$ In addition to mortality and morbidity, trauma places a huge financial burden on health systems. ${ }^{2}$ Multiple factors contribute to the trauma epidemic, including poverty, substance abuse, alcohol abuse, and interpersonal violence. ${ }^{1}$ The most significant factor contributing to trauma-related morbidity and mortality is the severity of injuries sustained. ${ }^{3}$ Various scoring systems have subsequently been developed in an attempt to objectively estimate the risk of trauma-related mortality. Trauma scoring systems can be categorised into three groups: i) Scores based on anatomical descriptions of injuries (e.g. abbreviated injury score (AIS), injury severity score (ISS) $)^{4}$; ii) Scores based on physiological parameters of injured patients (e.g. revised trauma score (RTS), Glasgow coma scale (GCS) $)^{4}$; and iii) Scores that use a combination of anatomical descriptions and physiological parameters (e.g. trauma and injury severity score (TRISS), ${ }^{4}$ Kampala trauma score $(\mathrm{KTS})^{5}$ ).

The accuracy of trauma scores has been previously compared, with different results depending on the outcome used. For example, the ISS predicted hospitalisation better than TRISS and RTS, ${ }^{6}$ while the ISS also performed well in predicting mortality in patients with firearm injuries. ${ }^{7}$ In Ghana, the KTS performed adequately to predict mortality and need for hospitalisation. ${ }^{5}$ 
All trauma scoring systems have certain limitations, and none of them is ideal for every setting. The ISS and the RTS are widely used in trauma registries in high-income countries, while the KTS has specifically been developed for low-income countries with severe resource limitations, as it is a simple score that does not require many parameters. ${ }^{8}$ No study has been conducted in South Africa comparing different trauma scores as predictors of mortality at the district healthcare level. Identifying the most appropriate trauma score will assist to objectively predict mortality and morbidity risk in trauma patients and might therefore assist with early identification of patients who need prompt referral to facilities able to provide a higher level of care. This should hopefully decrease the time to definitive care and potentially limit morbidity and mortality. The objective of this study was to compare the performance of four trauma scores (ISS, RTS, KTS, and TRISS) in predicting mortality in trauma patients managed within the resuscitation unit of a district-level hospital in Cape Town, South Africa.

\section{Methods}

\section{Study design}

A nested diagnostic test accuracy study within a retrospective cross-sectional study of a prospectively collected database was performed. The study was approved by the Stellenbosch University Health Research Ethics Committee (Ref: S16/08/147).

\section{Study setting and population}

Khayelitsha Hospital is a 300-bed large urban district-level hospital located in the township of Khayelitsha, one of the biggest townships of Cape Town in the Western Cape. The population is predominantly black with a high unemployment rate. ${ }^{2}$ The population of Khayelitsha is not spared by the quadruple burden of diseases affecting South Africa, that includes HIV/AIDS and tuberculosis, non-communicable diseases, maternal and child mortality and trauma. ${ }^{10}$ Different specialised services are rendered at Khayelitsha Hospital including medical, paediatrics, surgical, orthopaedics, and obstetrics and gynaecology. Khayelitsha Hospital has a large emergency centre, with a resuscitation unit that has four beds and one cot for paediatrics cases. Each bed is equipped with a ventilator and a resuscitation trolley.

The Khayelitsha Hospital Emergency Centre database was established on 1 November 2014 and is registered at the Stellenbosch University Health Research Ethics committee (Ref: N15/10/107). It is an observational electronic database that prospectively collects data on all patients managed within the resuscitation unit of Khayelitsha Hospital; the exact methodology has been previously described. ${ }^{9}$

\section{Data collection and management}

All trauma patients managed in the resuscitation unit were extracted from the database for a six-month period (1 November 2014 to 30 April 2015). Data not yet captured or missing were retrospectively added after scrutinising the clinical notes. Incomplete data points were excluded from the relevant analysis and were indicated as such.
Collected variables included patients demographics (age, gender), date and time of presentation, patient acuity, mechanism of injury, time spent in the resuscitation unit, and disposition from the resuscitation unit. The predictor variables were injury severity score (ISS), revised trauma score (RTS), Kampala trauma score (KTS), and trauma and injury severity score (TRISS). The outcome variable was all-cause in-hospital mortality, and includes trauma patients that died in Khayelitsha Hospital and those transferred to the referral hospital (Tygerberg Hospital) for higher level of care and subsequently died. The triage early warning score (TEWS) was used to determine patient acuity. The TEWS is a composite score of physiologic parameters measured at arrival to the hospital. It forms part of the South African triage scale (SATS) and categorizes patients as non-urgent (green), urgent (yellow), very urgent (orange), and emergency (red). ${ }^{11}$ We acknowledge that the TEWS is not validated to determine acuity in trauma patients, but was used to give an idea of the acuity relating to the patients' vital signs (the discriminator list of the SATS would have categorised all trauma patients at least as very urgent (orange)). Missing variables were retrospectively collected by a single investigator (SNM) and entered into a Microsoft Excel spreadsheet with validated functions to calculate each trauma score automatically. The collected data were not cross-checked due to logistical constraints. A detailed description of the calculation of the different trauma scores is available as supplementary material (Appendix1).

\section{Analysis}

Data were imported from Microsoft Excel (Microsoft Corp, Redmund WA) into STATA 14 (StataCorp, College Station, Texas) for analysis. Simple descriptive statistics were used to describe variables. Continuous variables were described using means and standard deviation (SD) or mean and inter-quartile range (IQR), where appropriate. Normality of variables was tested qualitatively and quantitatively. Diagnostic test accuracy analysis included only patients older than 14 years of age as the trauma scores are not validated in patients younger than 14 years. An empirical diagnostic cut-off point using receiver operating characteristics (ROC) curves to maximise the product of sensitivity and specificity for each trauma score

\begin{tabular}{|c|c|}
\hline & n (\%) \\
\hline \multicolumn{2}{|l|}{ Gender } \\
\hline Male & $726(83.6)$ \\
\hline Female & $142(16.4)$ \\
\hline \multicolumn{2}{|l|}{ Mechanism of injury } \\
\hline Mainly blunt & $376(43.3)$ \\
\hline Mainly penetrating & $492(56.7)$ \\
\hline \multicolumn{2}{|l|}{ Patient acuity* } \\
\hline Emergency / Red & $170(19.6)$ \\
\hline Very urgent / Orange & $256(29.5)$ \\
\hline Urgent / Yellow & $318(36.6)$ \\
\hline Non-urgent / Green & $123(14.2)$ \\
\hline
\end{tabular}


was used. Univariate logistic regression was used to determine significant associations and predictive values of triage scores.

A total of 918 trauma-related patients were extracted from the database for the 6-month study period. Only 868 patients were included in the study after 50 (5.4\%) patients were excluded due to missing records. The mean $( \pm \mathrm{SD})$ age of patients was $28 \pm 11$ years and $726(83.6 \%)$ were males. The acuity was at least very urgent (red and orange) in 426 (49.1\%) patients and penetrating trauma $(n=492,56.7 \%)$ was the prevailing mechanism of injury (Table 1). Patients spent a median (IQR) time in the resuscitation area of 151 minutes (90 to 241 minutes).

Overall, 45 (5.2\%) patients died, of which 40 (88.9\%) were males $(n=40,88.9 \%)$ and most had an acuity of at least very urgent $(n=42,93.4 \%)$. The mechanism of injury in patients who died was fairly evenly distributed between penetrating injuries $(\mathrm{n}=23,51.1 \%)$ and blunt injuries $(\mathrm{n}=22,48.9 \%)$. TRISS was the best predictor of mortality with an area under the ROC curve (AUC) of 0.93 (Table 2, Figure 1). If the estimates for TRISS at the empirical cut point of 0.96 are applied to a hypothetical cohort of 1000 trauma-related individuals, where $50(5 \%)$ of those actually die from their injuries, TRISS would be expected to miss five cases $(10 \%)$ and falsely predict death in 145 cases (15\%). Although TRISS outperformed the other scores, the differences were not statistically significant as evident by the overlapping confidence intervals. The individual ROC curves for each score are presented in Appendix 2.

\section{Discussion}

This is the first study comparing the diagnostic performance of trauma scores in a district-level hospital in South Africa. Patients were typically young males with an equal spread between penetrating and blunt mechanism of injury. The trauma and injury severity score (TRISS) performed the best with regards to AUC, effect size and other diagnostics, although the difference was not statistically significant.

The equivalence of the diagnostic performances of the four trauma scores supports previous findings. Macleod et al. also indicated small differences between KTS, RTS, ISS and

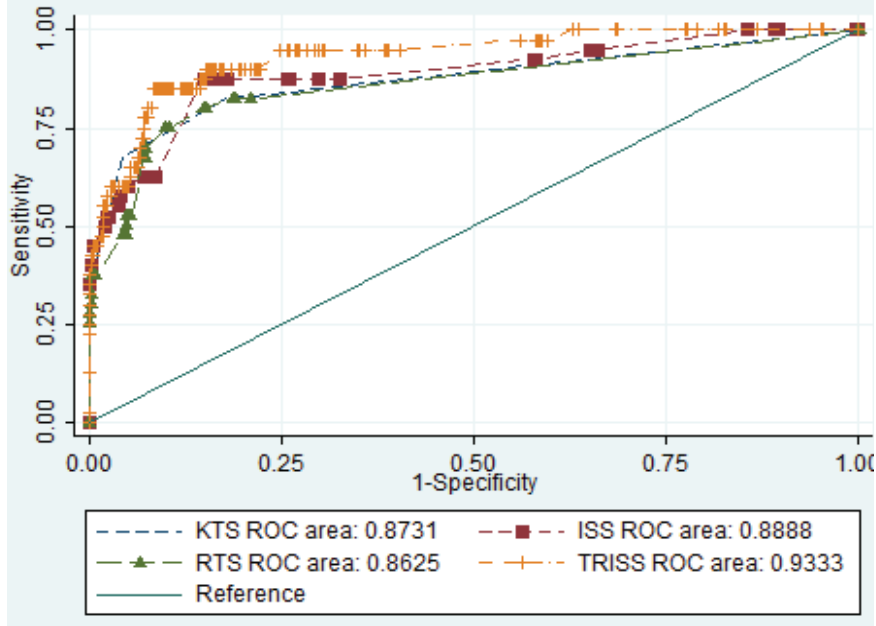

Figure 1. Receiver operator characteristics curves of the different trauma scores in predicting mortality in trauma-related patients presenting to the resuscitation unit of Khayelitsha Hospital.

TRISS. ${ }^{12}$ If none of the trauma severity scores is superior with regards to diagnostic performance, one can then rely on other factors to decide on the most appropriate score to implement. One argument is that scores need to be user friendly, practical and easy to calculate, ${ }^{5}$ e.g. KTS is much easier to use than ISS. On the other hand, with modern day applications available on mobile devices (even when offline), complicated equations should not be much of a hindrance.

Trauma scoring systems can be used for indications other than prognostic purposes. They may be used as a triage tool to improve the timely detection of patients that need prompt referral to higher level of care, although they still need to be tested formally. ${ }^{12}$ One would then hope that the triage system already in use performs adequately. Another viewpoint is to persist with the system already in use, as the use of different tools would certainly lead to confusion and medical errors. ${ }^{13}$ The South African triage scale is the preferred triage tool in Khayelitsha Hospital (and the Western Cape) and a direct comparative study is needed to identify the most appropriate tool to be used locally.

The majority of the study population were young male patients. This is typical of trauma-related patients as described

Table 2. Diagnostic accuracy of trauma scores in predicting mortality of trauma-related patients managed within the resuscitation unit of Khayelitsha Hospital

\begin{tabular}{|c|c|c|c|c|c|c|c|c|}
\hline $\begin{array}{l}\text { Trauma } \\
\text { score }\end{array}$ & Range & $\begin{array}{l}\text { Empirical } \\
\text { cut-point }\end{array}$ & $\mathbf{A U C *}$ & $\begin{array}{c}\text { Odds Ratio } \\
\left(95 \% \mathrm{CI}^{\dagger}\right)\end{array}$ & $\begin{array}{l}\text { Sensitivity } \\
(95 \% \text { CI })\end{array}$ & $\begin{array}{l}\text { Specificity } \\
(95 \% \text { CI) }\end{array}$ & $\begin{array}{c}\text { PPV } \\
(95 \% \text { CI })\end{array}$ & $\mathrm{NPV}^{\S}(95 \% \mathrm{CI})$ \\
\hline TRISS $₫$ & $0-1$ & 0.96 & 0.93 & $\begin{array}{c}53.9 \\
(19.7-147)\end{array}$ & $\begin{array}{c}90.7 \\
(77.9-97.4)\end{array}$ & $\begin{array}{c}84.7 \\
(81.9-87.1)\end{array}$ & $\begin{array}{c}24.8 \\
(18.3-32.4)\end{array}$ & $\begin{array}{c}99.4 \\
(98.4-99.8)\end{array}$ \\
\hline ISS $^{\|}$ & $1-75$ & 24.5 & 0.89 & $\begin{array}{c}40 \\
(15-101)\end{array}$ & $\begin{array}{c}87.5 \\
(73.2-95.8)\end{array}$ & $\begin{array}{c}85.1 \\
(82.4-87.5)\end{array}$ & $\begin{array}{c}23.5 \\
(16.9-31.1)\end{array}$ & $\begin{array}{c}99.2 \\
(98.2-99.8)\end{array}$ \\
\hline KTS $^{* *}$ & $5-16$ & 12.5 & 0.88 & $\begin{array}{c}23.8 \\
(10.6-53.4)\end{array}$ & $\begin{array}{c}83.7 \\
(69.3-93.2)\end{array}$ & $\begin{array}{c}82.2 \\
(79.3-84.8)\end{array}$ & $\begin{array}{c}20.8 \\
(15-27.6)\end{array}$ & $\begin{array}{c}98.9 \\
(97.8-99.6)\end{array}$ \\
\hline RTS $^{\dagger \dagger}$ & $0-10$ & 7.2 & 0.87 & $\begin{array}{c}22.2 \\
(9.9-49.8)\end{array}$ & $\begin{array}{c}83.7 \\
(69.3-93.2)\end{array}$ & $\begin{array}{c}81.2 \\
(78.2-83.9)\end{array}$ & $\begin{array}{c}19.9 \\
(14.3-26.5)\end{array}$ & $\begin{array}{c}98.9 \\
(97.7-99.6)\end{array}$ \\
\hline
\end{tabular}

*Area under receiver operator characteristics curve; †Confidence interval; $\$$ Positive predictive value; $\S$ Negative predictive value; $\uparrow$ Trauma and injury severity score; $\|$ Injury severity score; **Kampala trauma score, $\dagger \dagger$ Revised trauma score 
before. ${ }^{14}$ However, the predominance of penetrating injuries differs from international studies, where more blunt injuries are seen,${ }^{14}$ yet is characteristic of the South African trauma burden.

The all-cause in-hospital mortality was almost double compared to an Indian study. ${ }^{14}$ The exact reason for this was beyond the scope of this study, but could possibly relate to the high acuity of patients managed or that definitive surgical care at Khayelitsha Hospital was limited at the time of the study. The mechanism of injury in patients who died was almost equally distributed between blunt and penetrating injuries contrary to another study where more patients died from blunt injuries. ${ }^{15}$

The study has various limitations and care should be taken to generalise the findings. Firstly, the low mortality rate limits the validity of the results as it is evident in the wide confidence intervals. Secondly, although missing data were limited by including a chart review, missing or limited details inevitably incorporate an inherent risk of error. More than 5\% of cases were missing and, since it is impossible to determine their specific outcomes, the direction in which the results would have been influenced remains unknown. Thirdly, the study was conducted at one hospital with a small sample size for a short period of six months; this limits significantly the generalisability of the findings. Lastly, the study was only performed in a single district-level hospital due to logistical constraints. It further reflects a population with a high percentage of penetrating injuries that might be completely different to other settings.

\section{Conclusion}

The ISS, RTS, KTS and TRISS performed equivocally in predicting mortality in trauma-related patients managed in the resuscitation unit of Khayelitsha Hospital. Multicentred and large-scale studies are needed to confirm the results. The performance of trauma scores in the district-level hospital setting of South Africa needs further exploration.

\section{Acknowledgements}

I would like to express my appreciation to Dr DJ van Hoving for his patience, supervision and guidance during the study. I would also like to thank Michael McCaul for the statistics analysis. Lastly, thanks to Khayelitsha Hospital, especially Dr Sa'ad Lahri, who allowed me to collect data.

\section{Declaration}

By submitting this dissertation electronically, I, Suzan Nyema Mukonkole, declare that the entirety of the work contained therein is my own original work, that I am the authorship owner thereof (unless to the extent explicitly otherwise stated), and that I have not previously in its entirety or in part, submitted it for obtaining any qualification.

\section{REFERENCES}

1. Statistics South Africa. Mortality and causes of death in South Africa, 2012: Findings from death notification. Pretoria: Statistics South Africa, 2014. Available from: http://beta2.
statssa.gov.za/publications/P03093/P030932012.pdf Accessed 11 December 2015

2. Wesson HKH, Boikhutso N, Bachani AM, et al. The cost of injury and trauma care in low- and middle-income countries: a review of economic evidence. Health Policy Plan 2014;29(6):795-808. Available from: https://doi.org/10.1093/ heapol/czt064 PMID: 24097794.

3. Haagsma JA, Graetz N, Bolliger I, et al. The global burden of injury: incidence, mortality, disability- adjusted life years and time trends from the Global Burden of Disease study 2013. Inj Prev. 2016;22(1):3-18. Available from: https://doi.org/10.1136/ injuryprev-2015-041616 PMID: 26635210.

4. Pohlman TH, Bjerke HS, Offner P. Trauma Scoring Systems. Available from: https://emedicine.medscape.com/ article/434076-overview. Accessed 25 November 2018

5. Kobusingye OC, Lett RR. Hospital-based trauma registries in Uganda. J Trauma. 2000;48(3):498-502. Available from: https://doi.org/10.1097/00005373-200003000-00022 PMID: 10744292.

6. Orhon R, Eren ŞH, Karadayı Ş, et al. Comparison of trauma scores for predicting mortality and morbidity on trauma patients. Ulus Travma Acil Cerrahi Derg. 2014;20(4):258-64. Available from: https://doi: 10.5505/tjtes.2014.22725 PMID: 25135020.

7. Köksal Ö, Özdemir F, Bulut M,et al. Comparison of trauma scoring systems for predicting mortality in firearm injuries. Ulus Travma ve Acil Cerrahi Derg. 2009;15(6):559-64. PMID: 20037873.

8. Weeks SR, Juillard CJ, Monono ME, et al. Is the Kampala Trauma Score an effective predictor of mortality in low-resource settings? A comparison of multiple trauma severity scores. World J Surg. 2014;38(8):1905-11. Available from: https://doi. org/10.1007/s00268-014-2496-0 PMID: 24715042.

9. Hunter LD, Lahri S, van Hoving DJ. Case mix of patients managed in the resuscitation area of a district-level public hospital in Cape Town. Afr J Emerg Med. 2017;7(1):19-23. Available from: https://doi.org/10.1016/j.afjem.2017.01.001 PMID: 30485864.

10. Coovadia H, Jewkes R, Barron P, Sanders D, Mcintyre D. The health and health system of South Africa: historical roots of current public health challenges. Lancet 2009;374(9692):81734. Available from: https://doi.org/10.1016/S01406736(09)60951-X PMID: 1970972.

11. Twomey M, Wallis L, Thompson M, et al. The South African Triage Scale (adult version) provides reliable acuity ratings. Int Emerg Nurs. 2012;20(3):142-50. Available from: https://doi. org/10.1016/j.ienj.2011.08.002 PMID: 22726946.

12. MacLeod JBA, Kobusingye O, Frost C, et al. A Comparison of the Kampala Trauma Score (KTS) with the Revised Trauma Score (RTS), Injury Severity Score (ISS) and the TRISS Method in a Ugandan Trauma Registry. Eur J Trauma. 2003;29(6): 3928. Available from: https://doi.org/10.1007/s00068-003-1277-5

13. Bruijns SR, Wallis LA. The Kampala Trauma Score has poor diagnostic accuracy for most emergency presentations. Injury. 2017;48(10):2366-7. Available from: https://doi.org/10.1016/j. injury.2017.07.032 PMID:28855083.

14. Abhilash KP, Chakraborthy N, Pandian GR, et al. Profile of trauma patients in the emergency department of a tertiary care hospital in South India. J Fam Med Prim Care. 2016;5(3):55863. Available from: https://doi.org/10.4103/2249-4863.197279 PMID: 28217583.

15. Moodley NB, Clarke D, Aldous C. An audit of trauma-related mortality in a provincial capital in South Africa. S Afr J Surg. 2014;52(4):101-4. Available from: https://doi.org/10.7196/ SAJS.1995 PMID 28876698 


\section{Appendix 1 - Calculation of the different trauma scores}

\section{Injury severity score}

\section{Abbreviated injury scale (AIS)}

The AIS is an anatomical scoring system first introduced in 1969. Since this time it has been revised and updated against survival so that it now provides a reasonably accurate ranking of the severity of injury.

The AIS describes the severity of injury to one body region on a scale of 1 to 6 , with 1 being minor, 5 severe and 6 a nonsurvivable injury. This represents the 'threat to life' associated with an injury and is not meant to represent a comprehensive measure of severity. The AIS is not an injury scale, in that the difference between AIS1 and AIS2 is not the same as that between AIS4 and AIS5.

\begin{tabular}{ll} 
AIS Score & Injury \\
\hline 1 & Minor \\
2 & Moderate \\
3 & Serious \\
4 & Severe \\
5 & Critical \\
6 & Non-survivable
\end{tabular}

\section{ISS calculation}

To calculate an ISS for an injured person, the body is divided into six regions. These body regions are:

- Head and neck, including cervical spine

- Face, including the facial skeleton, nose, mouth, eyes and ears

- Thorax, thoracic spine and diaphragm

- Abdomen, abdominal organs and lumbar spine

- Extremities including pelvic skeleton

- External soft tissue injury

An ISS is then calculated according to ISS $=\mathrm{A} 2+\mathrm{B} 2+\mathrm{C} 2$, where A, B, C are the AIS scores of the three most injured body regions. The ISS ranges from 0 to 75 (i.e. AIS scores of 5 for each category). If any of the three scores is a 6 , the score is automatically set at 75 . Since a score of 6 ("non-survivable") indicates the futility of further medical care in preserving life, this may mean a cessation of further care in triage for a patient with a score of 6 in any category.

\section{Revised trauma score}

Based on Glasgow coma scale, systolic blood pressure and respiratory rate.

$\begin{array}{llll}\text { Glasgow Coma } & \text { Systolic Blood } & \text { Respiratory } & \text { Coded } \\ \text { Scale (GCS) } & \text { Pressure (SBP) } & \text { Rate (RR) } & \text { Value } \\ 13-15 & >89 & 10-29 & 4 \\ 9-12 & 76-89 & >29 & 3 \\ 6-8 & 50-75 & 6-9 & 2 \\ 4-5 & 1-49 & 1-5 & 1 \\ 3 & 0 & 0 & 0\end{array}$

$\mathrm{RTS}=0.9368(\mathrm{GCS})+0.7326(\mathrm{SBP})+0.2908(\mathrm{RR})$
The RTS is heavily weighted towards the Glasgow coma scale to compensate for major head injury without multisystem injury or major physiological changes. Values for the RTS are in the range 0 to 7.8408. A decreasing score corresponds to a more severe injury. A threshold of RTS $<4$ has been proposed to identify those patients who should be treated in a trauma centre, although this value may be somewhat low.

\section{Trauma and injury severity score}

TRISS determines the probability of survival (Ps) of a patient from the ISS and RTS.

The formulae to use is Ps $=1 / 1(1+e-b)$ where ' $b$ ' is calculated from $b=b 0+b 1($ RTS $)+b 2$ (ISS) $+b 3$ (Age Index). Age index is 0 if the patient is below 54 years of age or 1 if 55 years and over.

b0 to b3 are coefficients which are different for blunt and penetrating trauma.

\begin{tabular}{lll} 
& Blunt & Penetrating \\
\hline b0 & -0.4499 & -2.5355 \\
b1 & 0.8085 & 0.9934 \\
b2 & -0.0835 & -0.0651 \\
b3 & -1.7430 & -1.1360
\end{tabular}

\section{Kampala trauma score}

The KTS closely resembles the TRISS and is a composite of ISS and RTS. Possible score range from 5 to 16 and as with the RTS a decreasing score corresponds to a more severe injury.

\begin{tabular}{ll} 
Variable & Score \\
\hline Age (years) & \\
$5-55$ & 2 \\
$<5$ or $>55$ & 1 \\
Systolic blood pressure (mmHg) & \\
$>89$ & 4 \\
$50-89$ & 3 \\
$1-49$ & 2 \\
Undetectable & 1 \\
Respiratory rate (/minute) & \\
$10-29$ & 3 \\
$>29$ & 2 \\
$<10$ & 1 \\
Neurological status & \\
Alert & 4 \\
Response to verbal stimuli & 3 \\
Response to painful stimuli & 2 \\
Unresponsive & \\
Serious injuries & \\
None & \\
One & \\
Two or more & \\
\hline Total score & 2 \\
\hline
\end{tabular}




\section{Appendix 2 - Individual receiver operator characteristics curves for each trauma score}

1. Receiver operator characteristics curve of trauma and injury severity score (TRISS) in predicting mortality in trauma-related patients presenting to the resuscitation unit of Khayelitsha Hospital, with area under the curve: 0.93

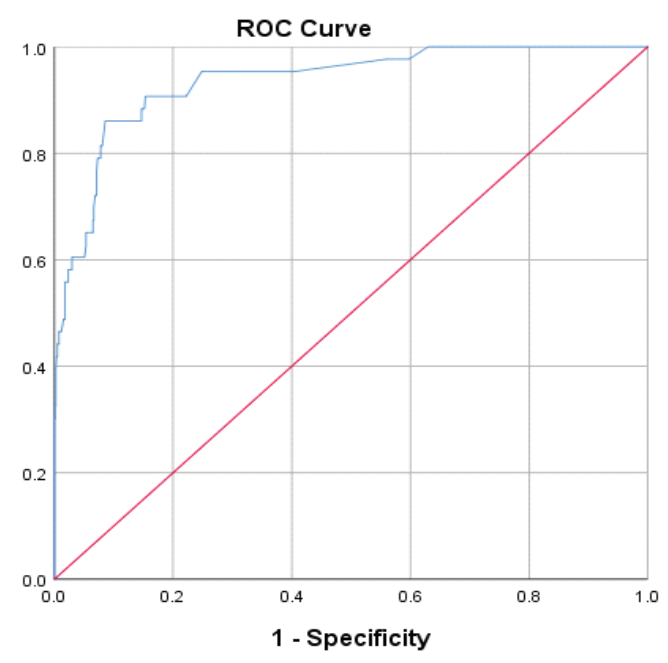

Diagonal segments are produced by ties

2. Receiver operator characteristics curve of injury severity score (ISS) in predicting mortality in trauma-related patients presenting to the resuscitation unit of Khayelitsha Hospital with area under the curve: 0.89

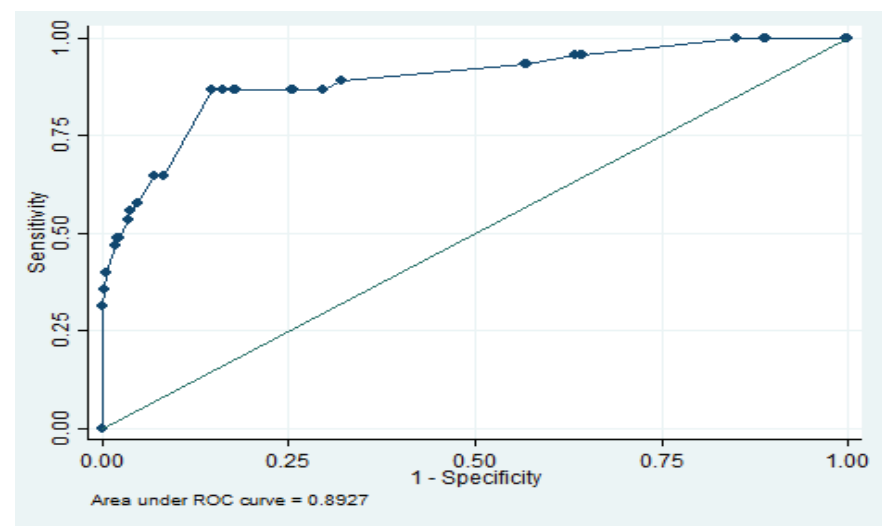

3. Receiver operator characteristics curve of Kampala trauma score (KTS) in predicting mortality in trauma-related patients presenting to the resuscitation unit of Khayelitsha Hospital with area under the curve: 0.88

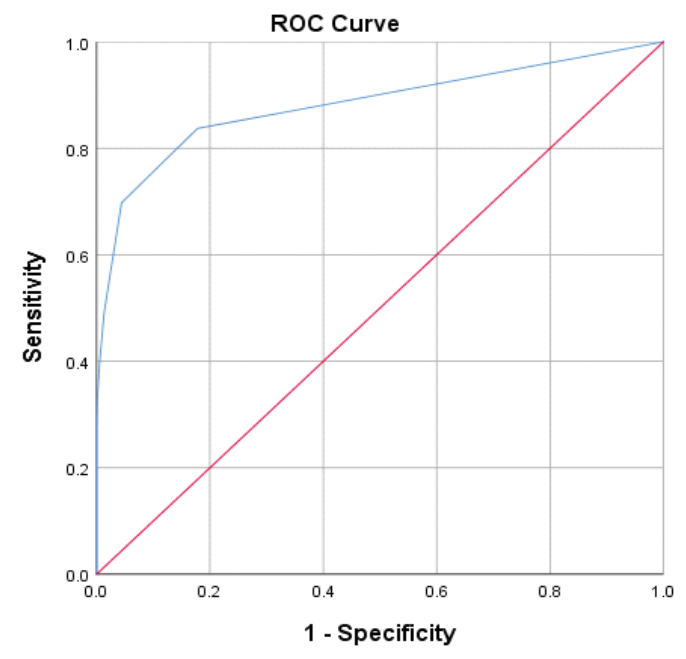

Diagonal segments are produced by ties.

4. Receiver operator characteristics curve of revised trauma score (RTS) in predicting mortality in trauma-related patients presenting to the resuscitation unit of Khayelitsha Hospital with area under the curve: 0.87

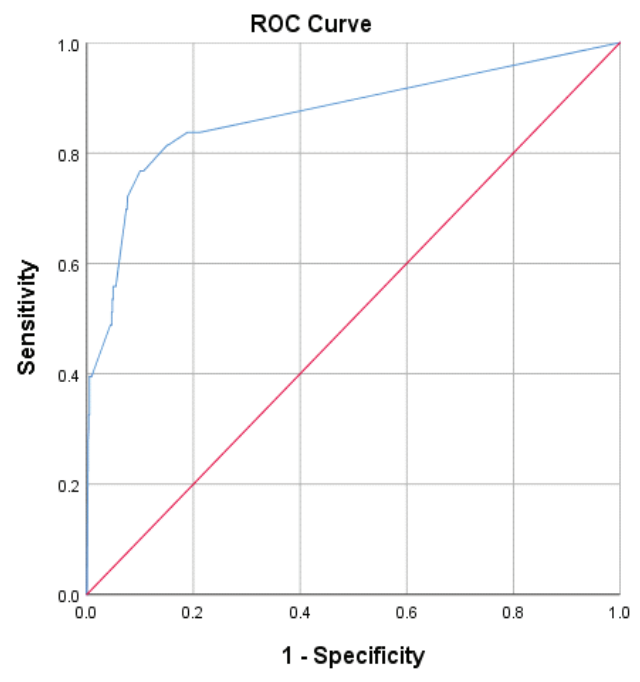

Diagonal segments are produced by ties. 